\title{
THE CONSTRAINED BOUNDARY ELEMENT METHOD, A TECHNIQUE ALLOWING GENERAL SURFACE MESHES IN MEMS SIMULATIONS
}

\author{
M. Bächtold and P. Ljung \\ Coyote Systems Inc., 2740 Van Ness Ave., San Francisco, USA \\ Phone: (415) 346-4223, Fax: (415) 346-6282, email: martinb@ coyotesystems.com
}

\begin{abstract}
The meshing of the device geometry is of critical importance in the numerical analysis of MEMS, since MEMS typically exhibit high geometric complexity and accurate simulation results require a well-chosen mesh. Most numerical methods for device simulation impose severe restrictions during the meshing process, accepting only very specific discretizations as legal computational meshes. For MEMS simulation models generated by an automated process [1], robust meshing and simulation tools are needed, capable of handling the generated geometries without user intervention. This work presents the Constrained Boundary Element Method (CBEM), an extension to the Boundary Element Method (BEM) [2], [3] which enables the robust analysis of 3D geometries by operating on general surface meshes.
\end{abstract}

\section{INTRODUCTION}

The meshing of a general 3D geometry for numerical analysis is a formidable task: numerical methods such as the finite elements (FEM), finite differences (FDM), boundary elements (BEM) or the box method (BM) impose a number of restrictions on the shape of the elements used and the geometric configurations allowed between neighboring elements. If these meshing restrictions are not satisfied valid results cannot be expected from a numerical analysis. Moreover, the discretization of a device geometry has a major impact on the accuracy and the computational cost of a simulation. A legal computational mesh can produce inaccurate results or cause prohibitive computational effort if the distribution of the elements does not conform to the distribution of the calculated solution. Ideally, a computational mesh should be anisotropically refined and should employ elements of different size, shape and polynomial order to optimally resolve the distribution of the computed solution fields [4]. Constructing such a mesh manually is a time-consuming task, requiring experience in numerical simulation to be able to estimate the accuracy of an obtained solution. MEMS designers want to be able to numerically analyze device designs quickly and accurately, without having to focus on meshing issues. This requires robust, sophisticated automated meshing tools and numerical methods which support very general mesh configurations.

Boundary integral methods such as the BEM only discretize material interfaces and contact surfaces [2], whereas volume-based methods like the FEM require a volumetric mesh of the entire device geometry. The ability of the BEM to operate on surface meshes is an important advantage for automated meshing, because it significantly simplifies the task of generating a valid computational mesh. However, manually constructing an optimal mesh is non-trivial and time-consuming, even when using boundary elements. This is especially true when dealing with anisotropic local mesh refinement and when mixing elements of various polynomial orders.

Local anisotropic mesh refinement, also known as h-type refinement, dramatically increases the accuracy of a simulation by introducing new elements exactly at those locations, where they are most needed to decrease the error in the computed solution distribution. However, anisotropic mesh refinement, as shown in Figure 1,

Anisotropic local refinement near to a corner of the structure

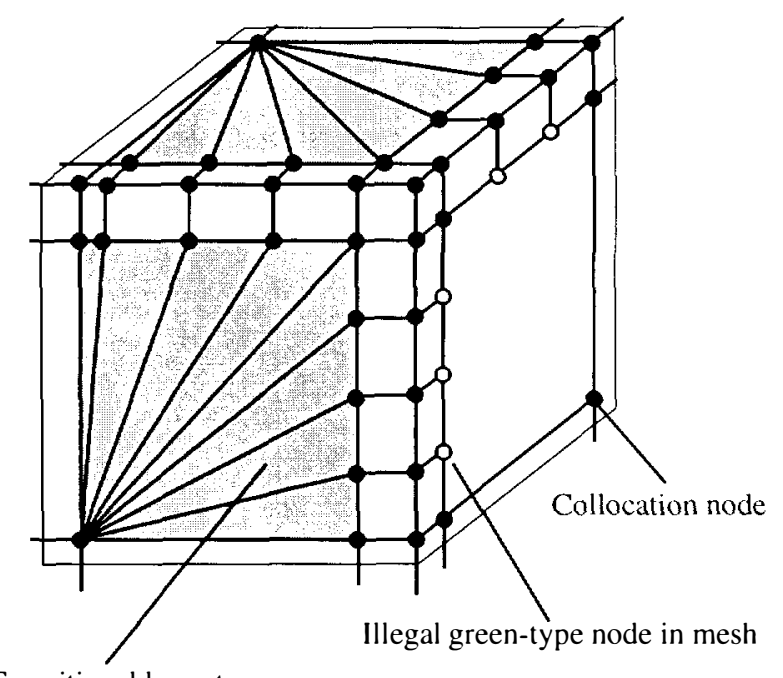

Transitional layer to

accommodate refinement

Figure 1. Meshing problems involved with h-type refinement and green-type nodes.

can introduce green-type nodes into the mesh, i.e., nodes which do not coincide an element vertex, but are located on the edge of another element. Such green-type nodes raise a number of meshing problems:

- Green-type nodes are illegal in most numerical methods, including FEM, and continuous BEM.

- Local refinement requires a transitional layer of elements to avoid green-type nodes (see Figure 1).

- The transitional elements cause large computational overhead.

- The transitional elements are often of bad shape (high aspect ratio) thereby affecting the overall solution accuracy

Other discretization problems arise when combining elements of different polynomial orders. As in the case of h-type refinement the solution accuracy can be significantly improved by using higher order elements in critical areas, a strategy also known 
TABLE 1: THE CONSTRAINED BOUNDARY ELEMENT METHOD

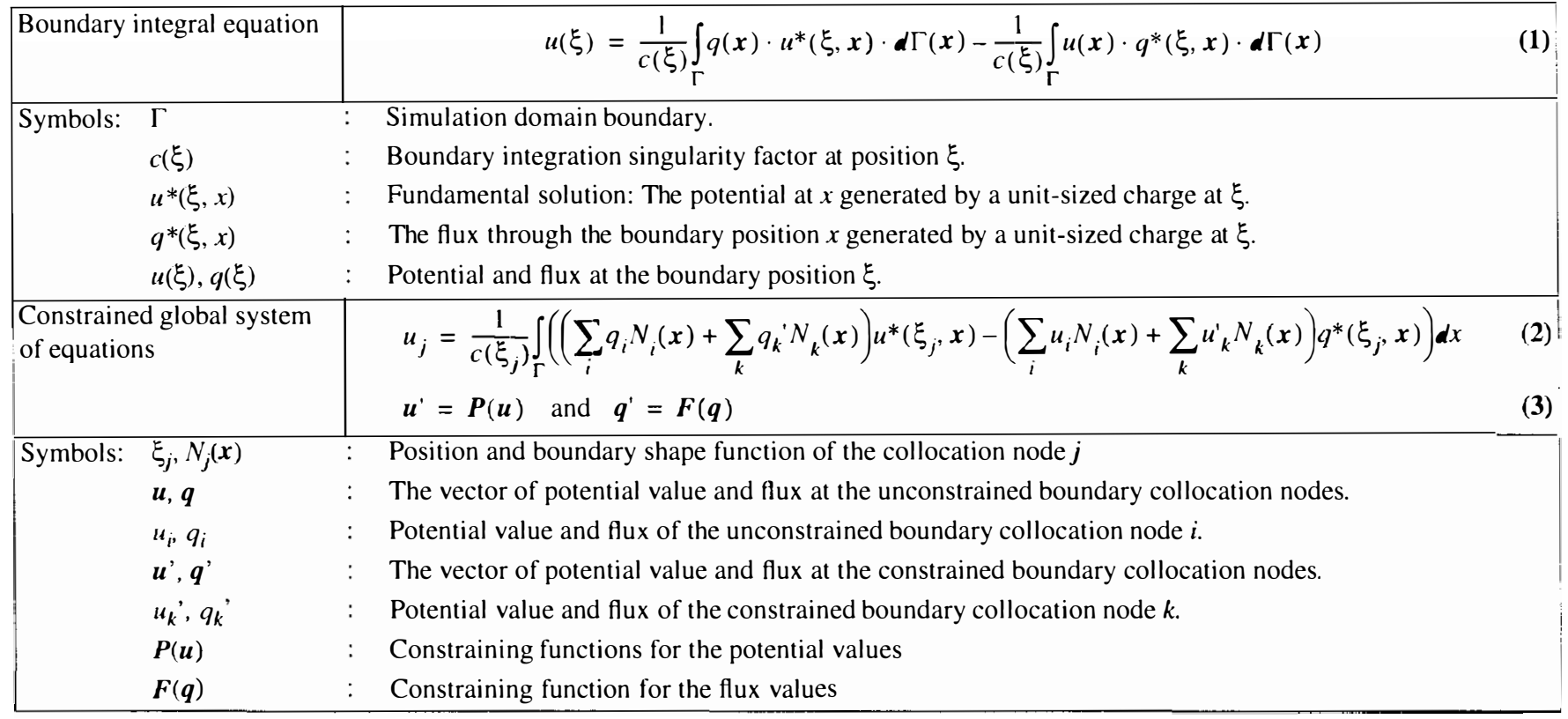

as p-type refinement (see Figure 2). The problems with p-type refinement in the meshing process include:

- To be able to apply local p-type refinement, various order elements must be able to mix freely in the mesh.

- Refining an element by increasing its order should not cause refinement in all its neighbor elements.

- Traditional numerical methods require many different element types to accommodate transitions between neighboring elements of varying order.

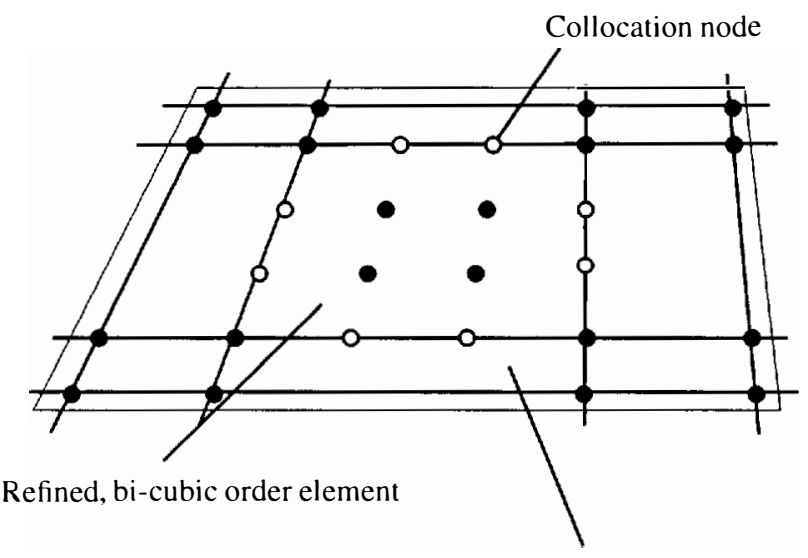

Special element for transition between different order elements

Figure 2. Meshing issues involved with p-type refinement when mixing elements of varying order.

This work presents the Constrained Boundary Element Method (CBEM), an approach which enables the robust analysis of 3D geometries while operating on general surface meshes. The CBEM facilitates the discretization process in two ways: being a boundary integral method, only interfaces instead of volumes are meshed, and general geometrical configurations of the elements are allowed by using constrained nodes. The CBEM is an enabling technology for automated device simulation, allowing numerical analysis directly from the mask layout of a device [1]. Further, the CBEM supports robust adaptive and anisotropic mesh refinement to construct an optimal mesh that delivers high solution accuracy with minimal computational effort [4].

\section{THE CONSTRAINED BOUNDARY ELEMENT METHOD}

The Boundary Element Method (BEM) solves the partial differential equation (PDE) that governs the device behavior by solving a discretized boundary integral formulation see equation (1) in Table 1. The BEM for continuous fields requires that the boundaries and material interfaces are meshed such that no green-type nodes occur and that neighboring elements are of the same order. This makes the construction of an initial mesh for an arbitrary geometry very difficult and also complicates subsequent mesh improvement using h-type or p-type refinement. In contrast, the CBEM handles green-type nodes and mixed order element edges by treating them as constrained. At such constrained nodes, the solution value is not found using the BEM's boundary integral formulation (equation (1)). The constrained node uses a constraint equation, as shown in equation (3), to compute its solution by interpolating the node's value from the solution values at the unconstrained nodes. The constrained node and the corresponding constraint equation at a green-type node are shown in Figure 3. Similarly, Figure 4 shows the constraint situation that arises when two neighboring elements are of different order.

The CBEM automatically identifies constrained nodes by checking for overlapping element edges at node positions. If overlapping edges are detected, the CBEM will determine for each node whether it should be marked as free or as constrained and supply 


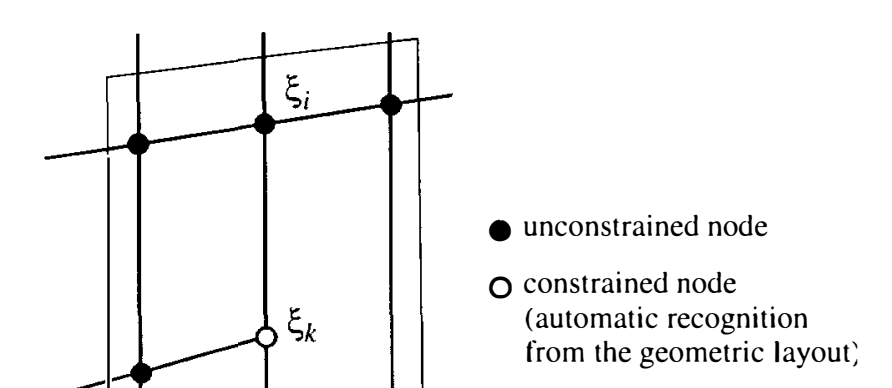

Constraining equations of $\xi_{k}$ $u_{k}=P_{k}\left(u_{i}, u_{j}\right)=0.5\left(u_{i}+u_{j}\right)$

$q_{k}=F_{k}\left(q_{i}, q_{j}\right)=0.5\left(q_{i}+q_{j}\right)$

Figure 3. Legal CBEM mesh containing h-type refinement using a constrained green-type node

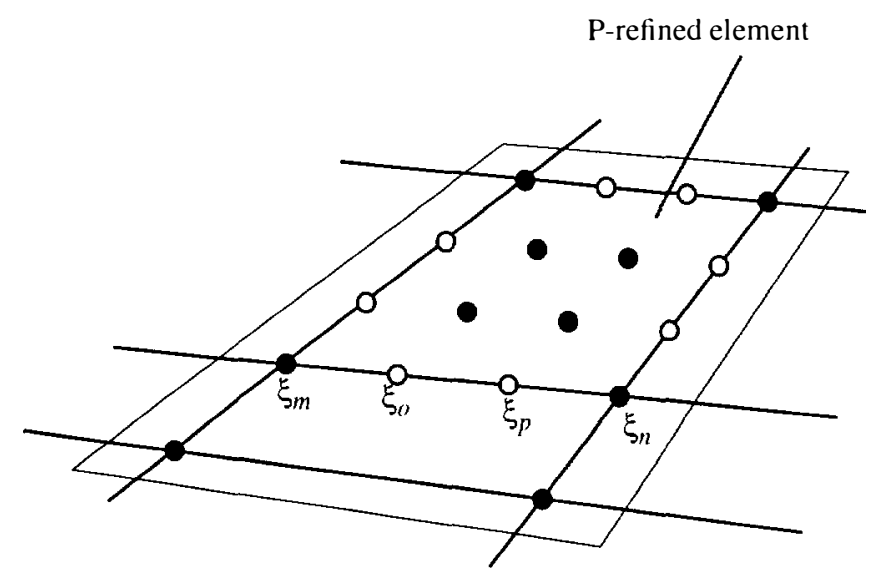

Constraining equations of $\xi_{o}$ $u_{o}=P_{o}\left(u_{m}, u_{n}\right)=0.67 u_{m}+0.33 u_{n}$ $q_{o}=F_{o}\left(q_{m}, q_{n}\right)=0.67 q_{m}+0.33 q_{n}$

Figure 4. Legal CBEM mesh containing a p-refined bi-cubic element, embedded in bi-linear elements

the corresponding constraining equation for each constrained node. The computational cost of recognizing constrained nodes in an arbitrary mesh is significantly reduced by using domain decomposition methods. Due to the linear nature of interpolation functions used in the constraint equations, it is possible to rewrite the set of constraint equations (3) as linear equation systems that allow determining the values at the constrained nodes from the solution at the unconstrained nodes:

$$
\boldsymbol{P}^{\prime} \cdot \boldsymbol{u}^{\prime}=\boldsymbol{P} \cdot \boldsymbol{u} \text { and } \boldsymbol{F}^{\prime} \cdot \boldsymbol{q}^{\prime}=\boldsymbol{F} \cdot \boldsymbol{q} .
$$

Here, the matrices $\boldsymbol{P}, \boldsymbol{P}, \boldsymbol{F}$, and $\boldsymbol{F}^{\prime}$ are extremely sparse matrices that contain the interpolation coefficients of the constraint equations shown in the Figures 3 and 4 . It is possible to sort the constraint variables such that the value with index $k$ depends only on the values at the unconstrained nodes and the constrained values with the indices $0 . . . k-1$ :

$$
u_{k}^{\prime}=P_{k}\left(u_{1 \ldots N}, u_{1 \ldots k-1}^{\prime}\right)
$$

with a similar formulation for the constrained flux variables $q_{k}$ '. This implies that the matrices $\boldsymbol{P}^{\prime}$ and $\boldsymbol{F}^{\prime}$ contain non-zero entries only on or below the main diagonal, thereby allowing us to efficiently solve for $\boldsymbol{u}^{\prime}$ and $\boldsymbol{q}$ ' in equation (4) by simple backsubstitution. Together, the BEM equations (equation (2) in Table 1) and the set of constraint equations (4) uniquely define the solution at all boundary nodes. This system of equations is solved efficiently using a matrix-free, iterative solver [5], shown in Figure 5. This keeps the computational cost of a constrained node constant, both in terms of run-time and memory consumption, regardless of the overall size of a simulation.

A finite element approach using constrained green-type nodes must carefully maintain flux conservation with the neighboring elements in order to converge to a valid solution of the governing PDE [6]. In contrast, the CBEM solution will always satisfy the PDE exactly, due to the fact that it is a superposition of Green's functions, so constrained nodes can easily be used.

\section{RESULTS}

The CBEM can robustly discretize any geometry generated using CAD tools, fabrication process emulation or imported from other sources. In Figure 6, the geometry of a MEMS accelerometer is imported and automatically discretized into a valid computational mesh for the CBEM. The initial mesh is then adaptively refined in several iterations, creating a highly optimized mesh to achieve high solution accuracy with minimal computational effort, as shown in Figure 7. The CBEM method readily integrates with BEM multipole acceleration techniques [7], [8] which require the matrix-free iterative solver shown in Figure 5. The flexibility of CBEM meshes and the efficiency of the multipole accelerated BEM allow MEMS

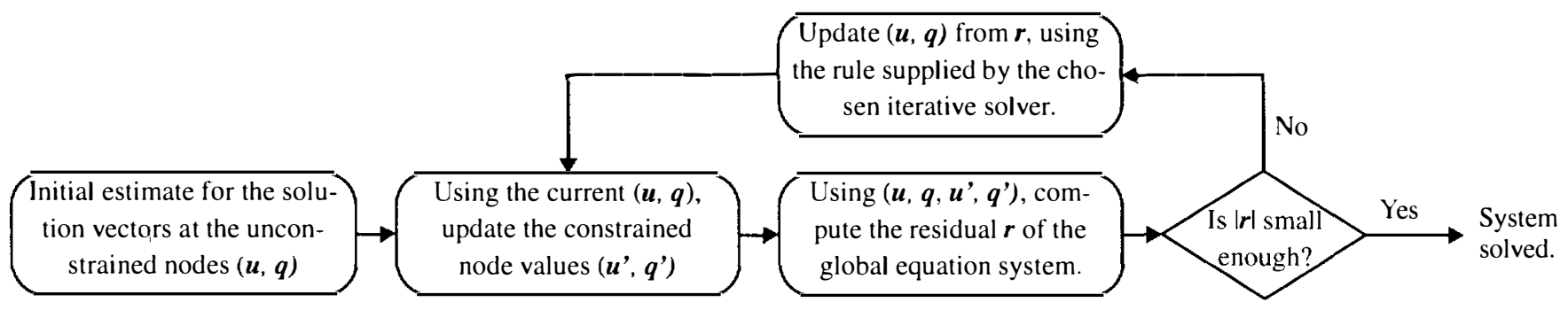

Figure 5. Solving the CBEM global system of equations using a matrix-free iterative method. Applicable are all Krylov subspace solvers, including the GMRES, CG, BiCG, CGS or Bi-CGSTAB methods [5]. 


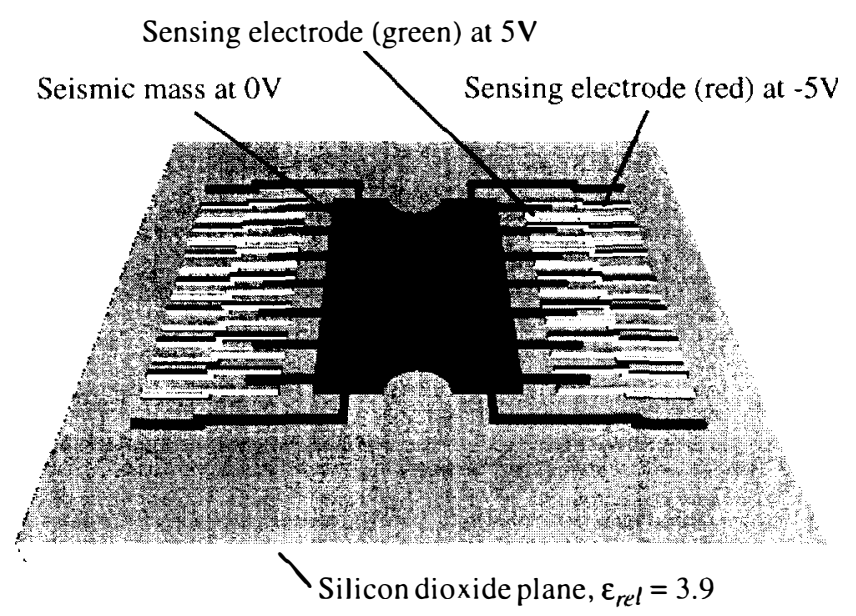

Figure 6. Automatically generated geometry of an electrostatic MEMS accelerometer

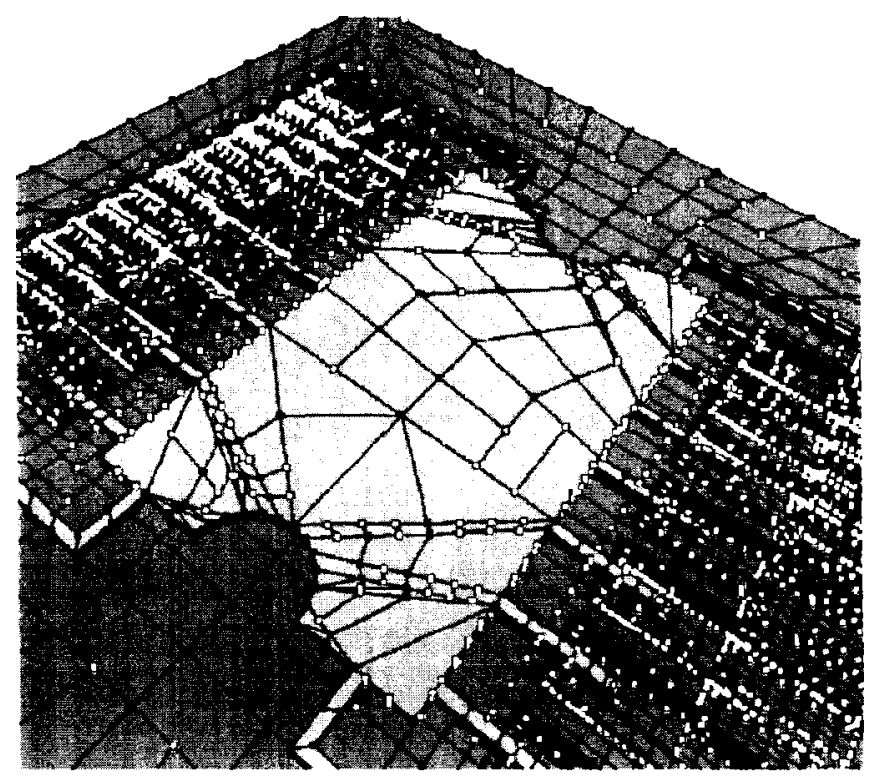

\begin{tabular}{|ll|}
\hline The adaptive CBEM mesh: \\
- 6 levels of refinement & - 2990 constrained nodes (white) \\
- 7125 elements & - 4663 unconstrained nodes (black)
\end{tabular}

Figure 7. CBEM mesh created from an automatically generated initial mesh using iterative adaptive refinement [4]

developers to simulate the behavior of an entire device instead of being limited to small portions of a complex system.

This CBEM model now has 4663 degrees of freedom (DOFs) and is solved in 10 minutes on an engineering workstation
(233 MHz Pentium II), using a multipole accelerated BEM engine [3]. The traditional BEM approach using discontinuous boundary elements [9] to handle the same general mesh and provide comparable accuracy requires 7,125 linear elements, resulting in 28,500 DOFs, and takes two hours to solve, when applying the same advanced multipole acceleration techniques.

\section{ACKNOWLEDGMENT}

This work is supported by DARPA under contract F3060296-2-0305.

\section{REFERENCES}

1. M. Bächtold, S. Taschini, J.G. Korvink, H Baltes, "Automated Extraction of Capacitances and Electrostatic Forces in MEMS and ULSI Interconnects from the Mask Layout", Proc. IEDM, IEEE, 1997, pp. 129-132.

2. P. Ljung, R. Howe, A. Pisano, "Sequential Solutions of Field Equations Using a Single BEM Model”, Tech. Digest Solid State Sensor and Actuator Workshop, Hiltonhead, 1996, pp. 117-122.

3. M. Bächtold, Efficient 3D Computation of Electrostatic Fields and Forces in Microsystems, Ph. D. Thesis, ETH Zurich, Physical Electronics Laboratory, 1997.

4. M. Bächtold, J.G. Korvink, H Baltes, "An Error Indicator and Automatic Adaptive Meshing for 3D Electrostatic Boundary Element Simulations", Boundary Elements XIX, Comput. Mechanics Publ., Southampton, 1997, pp. 709-718.

5. R. Barrett et al., Templates for the Solution of Linear Systems: Building Blocks for Iterative Methods, SIAM, Philadelphia PA, 1994.

6. J.G. Korvink, An Implementation of the Adaptive Finite Element Method for Semiconductor Sensor Simulation, Ph.D. thesis, ETH Zurich, Verlag der Fachvereine, Zurich, 1993.

7. K. Nabors, Efficient Three-Dimensional Capacitance Calculation, Ph.D. thesis, M.I.T., Cambridge MA, 1993.

8. M. Bächtold, J.G. Korvink, H Baltes, "Enhanced Multipole Acceleration Technique for the Solution of large Poisson Computations", IEEE Trans. on CAD of Integrated Circuits and Systems, vol. 15(12), 1996, pp. 1541-1546.

9. C.A. Brebbia, J.C.F. Telles, L.C. Wrobel, Boundary Element Techniques, Theory and Applications in Engineering, Springer Verlag Berlin, 1984. 